\title{
Predictive factors of para-aortic Iymph nodes metastasis in cervical cancer patients: a retrospective analysis based on 723 para-aortic lymphadenectomy cases
}

\author{
Xiaotian Han ${ }^{1,2, *}$, Hao Wen ${ }^{1,2, *}$, Xingzhu Ju ${ }^{1,2}$, Xiaojun Chen ${ }^{1,2}$, Guihao Ke ${ }^{1,2}$, Yuqi \\ Zhou $^{1,2}$, Jin Li ${ }^{1,2}$, Lingfang Xia ${ }^{1,2}$, Jia Tang ${ }^{1,2}$, Shanhui Liang ${ }^{1,2}$ and Xiaohua $\mathbf{W u}^{1,2}$ \\ ${ }^{1}$ Department of Gynecologic Oncology, Fudan University Shanghai Cancer Center, Shanghai, PR China \\ 2 Department of Oncology, Shanghai Medical College, Fudan University, Shanghai, PR China \\ * These authors have contributed equally to this work \\ Correspondence to: Xiaohua Wu, email: docwuxh@hotmail.com \\ Keywords: cervical cancer, para-aortic lymph node, pelvic lymph node, lymphadenectomy, SCCA \\ Received: October 07, $2016 \quad$ Accepted: February 21, $2017 \quad$ Published: March 08, 2017
}

Copyright: Han et al. This is an open-access article distributed under the terms of the Creative Commons Attribution License 3.0 (CC BY 3.0), which permits unrestricted use, distribution, and reproduction in any medium, provided the original author and source are credited.

\section{ABSTRACT}

Para-aortic lymph node (PALN) dissection is optional and controversial in patients with stage IB1-IIA2 cervical cancer. This retrospective study investigated PALN involvement patterns and evaluated preoperative clinical factors. A total of $\mathbf{7 2 3}$ consecutive FIGO stage IB1-IIA2 cervical cancer patients were included in the study. All patients underwent radical hysterectomy/radical trachelectomy, pelvic lymph node dissection, and PALN dissection. PALN metastasis was found in $101(\mathbf{1 4 . 0 \% )}$ patients, and the positive PALN rates of stage IB1, IB2, IIA1, and IIA2 were $8.4 \%$, $11.1 \%, 17.2 \%$ and $21.7 \%$, respectively. A multivariate model suggested age $>46$ years (OR: 1.67, 95\% confidence interval (CI): 1.08-2.58), tumor size $>3.5 \mathrm{~cm}$ (OR: 1.79, 95\% CI: 1.12-2.87), and FIGO stage IIA (vs. IB) (OR: 1.97, 95\% CI: 1.25-3.11) all positively correlated with PALN metastasis. When squamous cervical cancer cases were categorically analyzed, a multivariate model indicated age $>46$ years (OR: 1.67, 95\% CI: 1.00-2.80), FIGO stage IIA (vs. IB) (OR: 1.76, 95\% CI: 1.02-3.02), and squamous cell carcinoma antigen (SCCA) $>6.5 \mathrm{ng} / \mathrm{ml}$ (OR: 5.20, 95\% CI: 3.078.81) all positively correlated with PALN metastasis. Age, tumor size, and FIGO stage correlated with PALN metastasis in cervical cancer, while age, FIGO stage, and SCCA level were predictive in squamous cell carcinoma.

\section{INTRODUCTION}

The most recent data from GLOBOCAN 2012 showed the global incidence of cervical cancer was about 527,600 with an annual mortality of 265,700 [1]. Each year there are about 98,000 cervical cancer patients newly diagnosed in China [2], thus it is of great importance to optimize individual treatment of cervical cancer.

Lymph node (LN) metastasis is an independent prognostic factor for cervical cancer patients $[3,4]$. Typically, the adjacent obturator nodes will be the first site of LN metastasis, then it will spread in a step-wise fashion to the ipsilateral common iliac lymph nodes and para-aortic lymph nodes (PALN). Patients with PALN metastasis have lower survival rates and need extended field radiation. Dissection of enlarged PALN itself could have potential treatment value.

Pelvic lymphadenectomy is recommended by the National Comprehensive Cancer Network (NCCN) to treat cervical cancer [5]. However, the choice of paraaortic lymphadenectomy is usually optional because the incidence of PALN involvement in early stage cancer is low, and it is also difficult to diagnose positive PALN before surgery [6]. One recent meta-analysis concluded that CT and MRI had sensitivities of only $50 \%$ and $56 \%$, respectively. PET or PET-CT had much better accuracy with a sensitivity of $82 \%$ [7], but patients can have a $15 \%$ chance of positive PALN even with a negative PET-CT 
scan [8]. Moreover, PET-CT could not be widely-used in every patient due to cost efficiency, especially in developing countries.

To identify PALN metastasis predictive factors that can be evaluated before surgery, we conducted this retrospective study in patients with FIGO stage IB1-IIA2 cervical cancer, aiming to discover PALN involvement patterns and optimize the decision process of whether to perform PALN dissection.

\section{RESULTS}

\section{Patients' characteristics}

A total of 723 consecutive FIGO stage IB1-IIA2 cervical cancer patients who underwent pelvic and paraaortic lymphadenectomy were analyzed in this study. Median age of the population was 45 years (range: 20$76)$, and the minority of patients $(\mathrm{N}=177,24.5 \%)$ were in menopause. Squamous cell carcinoma was the dominating histological type with 639 cases $(88.4 \%)$. Among all these cases, the median number of PALNs removed was 4 (range: 1 to 41 nodes), $622(86.0 \%)$ patients had negative PALN, and the other $101(14.0 \%)$ had positive PALN. In 81 patients with small tumor size $(\leq 2.0 \mathrm{~cm})$, only 4 patients $(4.9 \%)$ had PALN metastasis. Ovarian metastasis was rare in our study, which only represented 12 cases: 6 with squamous cell cancer (SCC), 5 with adenocarcinoma (AC), and 1 with adenosquamous carcinoma (ACS).

Baseline demographic and clinical characteristics stratified by PALN metastasis are presented in Table 1. Based on best cutoff values of ROC curve, age, tumor size, and SCCA were respectively grouped by $>46 \mathrm{y},>3.5 \mathrm{~cm}$, and $>6.5 \mathrm{ng} / \mathrm{ml}$ in this study. Body mass index (BMI) was not obtained for two patients, the tumor size of 11 patients was not measureable, and SCCA was not detected in 105 $(16.4 \%)$ patients. The two groups did not differ in BMI, eastern cooperative oncology group (ECOG), menopause, childbirth, surgical type, and histological type. However, positive PALN group showed association in age $>46 \mathrm{y}$ ( $p$ $=0.007)$, advanced FIGO stage $(p=0.001)$, tumor size $>$ $3.5 \mathrm{~cm}(p=0.004)$, ovarian preservation $(p<0.001)$ and ovarian metastasis $(p=0.001)$.

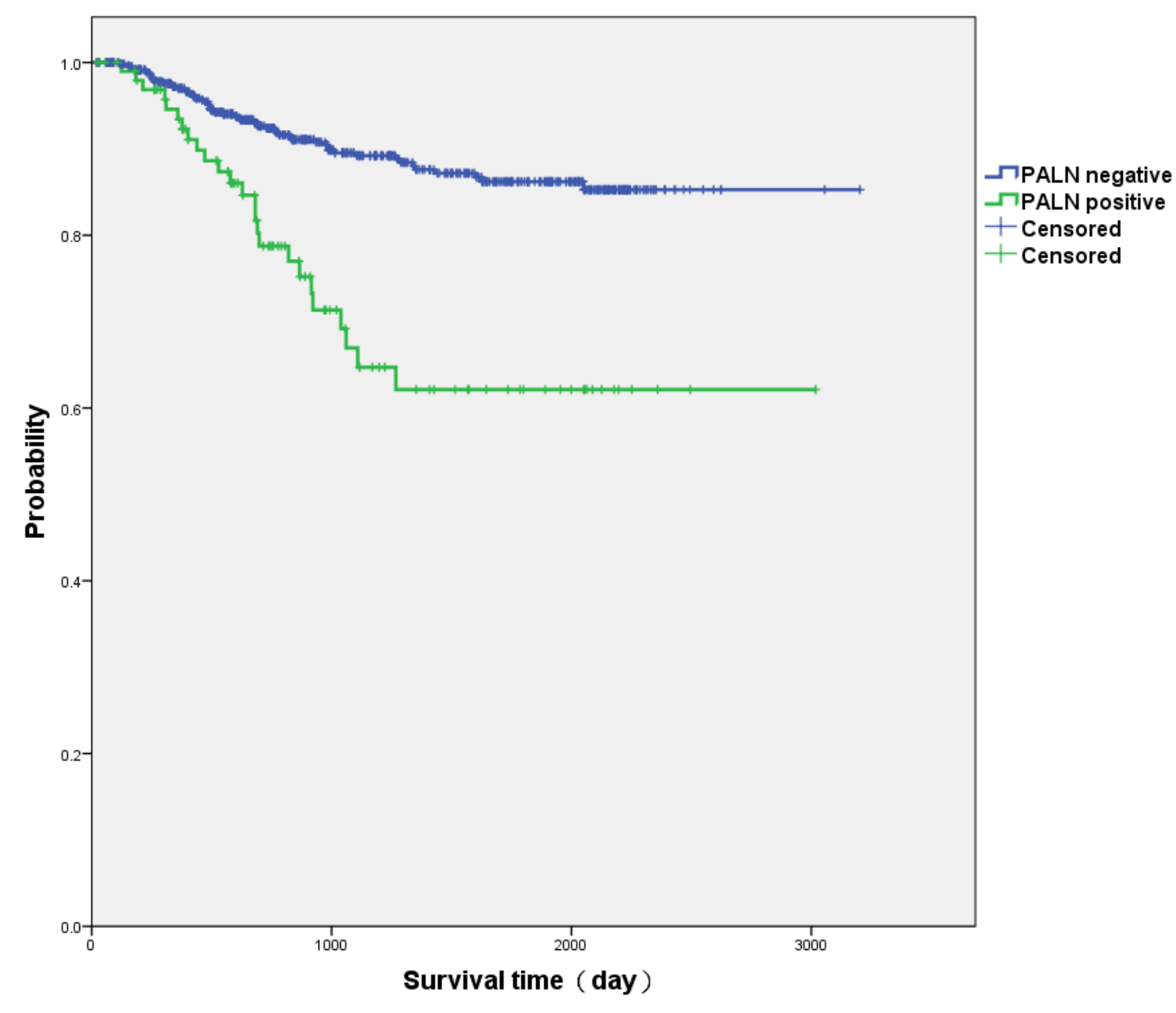

Figure 1: Kaplan-Meier curves of positive para-aortic lymph node (PALN) patients and negative PALN patients. Three-year survival rate of negative PALN patients was higher than that of positive PALN patients $(89.5 \pm 1.5 \% v s .67 .0 \pm 6.0 \%, p<0.001)$. 
Table 1: Baseline demographic and clinical characteristics of all patients

\begin{tabular}{|c|c|c|c|c|}
\hline \multicolumn{2}{|c|}{ Feature } & \multirow{2}{*}{$\frac{\text { PALN negative (\%) }}{622(100.0)}$} & \multirow{2}{*}{$\frac{\text { PALN positive (\%) }}{101(100.0)}$} & \multirow[t]{2}{*}{$p$ value } \\
\hline Total & & & & \\
\hline \multirow{2}{*}{ Age } & $<=46 y$ & $364(58.5)$ & $44(43.6)$ & \multirow{2}{*}{0.007} \\
\hline & $>46 y$ & $258(41.5)$ & $57(56.4)$ & \\
\hline \multirow{3}{*}{ BMI } & $<18.5$ & $50(8.1)$ & $7(6.9)$ & \multirow{3}{*}{0.841} \\
\hline & $18.5 \sim 24.9$ & $448(72.3)$ & $72(71.3)$ & \\
\hline & $>=25.0$ & $122(19.7)$ & $22(21.8)$ & \\
\hline \multirow{2}{*}{ ECOG } & 0 or 1 & $571(91.8)$ & $90(89.1)$ & \multirow{2}{*}{0.343} \\
\hline & 2 or 3 & $51(8.2)$ & 11(10.9) & \\
\hline \multirow{2}{*}{ Menopause } & No & $477(76.7)$ & $69(68.3)$ & \multirow{2}{*}{0.080} \\
\hline & Yes & $145(23.3)$ & $32(31.7)$ & \\
\hline \multirow{2}{*}{ Childbirth } & No & $33(5.3)$ & $3(3.0)$ & \multirow{2}{*}{0.459} \\
\hline & Yes & $589(94.7)$ & $98(97.0)$ & \\
\hline \multirow{4}{*}{ FIGO stage* } & IB1 & $252(40.5)$ & $23(22.8)$ & \multirow{4}{*}{0.001} \\
\hline & IB2 & $80(12.9)$ & $10(9.9)$ & \\
\hline & IIA1 & $178(28.6)$ & $37(36.6)$ & \\
\hline & IIA2 & $112(18.0)$ & $31(30.7)$ & \\
\hline \multirow{3}{*}{ Histological type } & $\mathrm{SCC}$ & $547(87.9)$ & $92(91.1)$ & \multirow{3}{*}{0.576} \\
\hline & $\mathrm{AC}$ & $49(7.9)$ & $5(5.0)$ & \\
\hline & ASC & $26(4.2)$ & $4(4.0)$ & \\
\hline \multirow{2}{*}{ Tumor size } & $<=3.5 \mathrm{~cm}$ & $271(44.3)$ & $29(29.0)$ & \multirow{2}{*}{0.004} \\
\hline & $>3.5 \mathrm{~cm}$ & $341(55.7)$ & $71(71.0)$ & \\
\hline \multirow{2}{*}{$\begin{array}{l}\text { Ovarian } \\
\text { preservation }\end{array}$} & No & $426(68.5)$ & $87(86.1)$ & \multirow{2}{*}{$<0.001$} \\
\hline & Yes & $196(31.5)$ & 14(13.9) & \\
\hline \multirow{2}{*}{ Ovarian metastasis } & No & $421(98.8)$ & $80(92.0)$ & \multirow{2}{*}{0.001} \\
\hline & Yes & $5(1.2)$ & $7(8.0)$ & \\
\hline
\end{tabular}

Abbreviations: PALN: para-aortic lymph node, BMI: body mass index, ECOG: eastern cooperative oncology group, SCC: squamous carcinoma, $\mathrm{AC}$ : adenocarcinoma, $\mathrm{ASC}$ : adenosquamous carcinoma

*FIGO stages are defined by the 2009 FIGO (International Federation of Gynaecology and Obstetrics) staging system.

The median follow-up time was 34.8 (31.3-38.3) months. Three-year survival rate of negative PALN patients was higher than that of positive PALN patients $(89.5 \pm 1.5 \%$ vs. $67.0 \pm 6.0 \%, p<0.001)$ (Kaplan-Meier curves in Figure 1).

\section{Lymph node metastasis pattern}

The number of cases of IB1, IB2, IIA1, and IIA2 stage cancers were $275,90,215$, and 143 , respectively (Table 2). The corresponding positive PALN rates were $8.4 \%, 11.1 \%, 17.2 \%$, and $21.7 \%$, which suggested that patients with more advanced FIGO stage were more likely to have PALN metastasis. PLN involvement rates also increased from $30.9 \%$ at the IB1 stage to $59.4 \%$ at the IIA2 stage.

Of 298 patients with positive PLN, 98 (32.9\%) had PALN metastasis at the same time. Positive PLN was associated with PALN in univariate logistic regression (OR: 68.93, 95\% CI: 21.59-220.10, $p<0.001$ ). Moreover, in 118 patients with positive common iliac LN, 77 patients $(65.3 \%)$ were also found positive for PALN metastasis.
Twenty-four patients with positive PALN showed no evidence of common iliac LN involvement, and 3 of 101 positive PALN patients (3.0\%) had no pelvic nodal disease. There was no uterine body involvement in the three skipped metastasis cases, only one of them had ovarian metastasis.

\section{Logistic analyses of PALN metastasis}

Univariate Logistic analyses suggested that age $>$ $46 \mathrm{y}$, post-menopausal, tumor size $>3.5 \mathrm{~cm}$, and FIGO stage IIA (vs. IB) were associated with higher risk of PALN metastasis $(p<0.1$; Table 3$)$. After a stepwise backward elimination of these 4 potential predictive factors in a multivariate model, 3 covariates remained significant: age > 46y (OR: 1.67, 95\% CI: 1.08-2.58; $p=$ 0.020 ), tumor size $>3.5 \mathrm{~cm}$ (OR: $1.79,95 \% \mathrm{CI}: 1.12-2.87$; $p=0.015)$ and FIGO stage IIA (vs. IB) (OR: $1.97,95 \%$ CI: $1.25-3.11 ; p=0.003)$. Positive PALN rate of patients with $0(n=119), 1(n=235), 2(n=257)$ and $3(n=112)$ of those risk factors were $5.0 \%, 9.4 \%, 17.5 \%$ and $25.0 \%$, respectively. 
Table 2: FIGO stage and lymph node metastasis rate

\begin{tabular}{|l|c|c|c|}
\hline \multicolumn{1}{|c|}{ FIGO stage } & Number & Positive PLN (\%) & Positive PALN (\%) \\
\hline IB1 & 275 & $85(30.9 \%)$ & $23(8.4 \%)$ \\
\hline IB2 & 90 & $38(42.2 \%)$ & $10(11.1 \%)$ \\
\hline IIA1 & 215 & $90(41.9 \%)$ & $37(17.2 \%)$ \\
\hline IIA2 & 143 & $85(59.4 \%)$ & $31(21.7 \%)$ \\
\hline Total & 723 & $298(41.2 \%)$ & $101(14.0 \%)$ \\
\hline
\end{tabular}

Abbreviations: FIGO: International Federation of Gynaecology and Obstetrics, PLN: pelvic lymph node, PALN: para-aortic lymph node

Table 3: Logistic regression of preoperative factors associated with para-aortic lymph node metastasis in cervical cancer patients $(N=723)$

\begin{tabular}{|c|c|c|c|c|c|}
\hline \multirow{2}{*}{\multicolumn{2}{|c|}{ Category }} & \multicolumn{2}{|c|}{ Univariate } & \multicolumn{2}{|c|}{ Multivariate } \\
\hline & & \multirow{2}{*}{$\frac{\text { OR (95\% CI) }}{1.83(1.20-2.79)}$} & \multirow{2}{*}{$\frac{P \text { value }}{0.005}$} & \multirow{2}{*}{$\begin{array}{c}\text { OR (95\% CI) } \\
1.67(1.08-2.58)\end{array}$} & \multirow{2}{*}{$\frac{P \text { value }}{0.020}$} \\
\hline Age (y) & $>46 v s .<=46$ & & & & \\
\hline Post-menopausal & Yes vs. No & $1.53(0.97-2.41)$ & 0.071 & $0.99(0.55-1.78)$ & 0.967 \\
\hline Childbirth & Yes vs. No & $1.83(0.55-6.08)$ & 0.324 & & \\
\hline Tumor size $(\mathrm{cm})$ & $>3.5$ vs. $<=3.5$ & $1.95(1.23-3.08)$ & 0.005 & $1.79(1.12-2.87)$ & 0.015 \\
\hline FIGO stage & IIA2+IIA1 vs. IB1+IB2 & $2.36(1.51-3.68)$ & $<0.001$ & $1.97(1.25-3.11)$ & 0.003 \\
\hline \multirow{2}{*}{ Histological type } & $\mathrm{AC} v s . \mathrm{SCC}$ & $0.61(0.24-1.56)$ & 0.301 & & \\
\hline & $\mathrm{ASC} v s . \mathrm{SCC}$ & $0.92(0.31-2.68)$ & 0.871 & & \\
\hline
\end{tabular}

Abbreviations: SCC: squamous carcinoma, AC: adenocarcinoma, ASC: adenosquamous carcinoma

Table 4: Logistic regression of preoperative factors associated with para-aortic lymph node metastasis in squamous cell carcinoma patients $(N=639)$

\begin{tabular}{|c|c|c|c|c|c|}
\hline \multirow{2}{*}{\multicolumn{2}{|c|}{ Category }} & \multicolumn{2}{|c|}{ Univariate } & \multicolumn{2}{|c|}{ Multivariate } \\
\hline & & OR $(95 \% \mathrm{CI})$ & $P$ value & OR $(95 \% \mathrm{CI})$ & $P$ value \\
\hline Age (y) & $>46$ vs. $<=46$ & $1.68(1.07-2.62)$ & 0.022 & $1.67(1.00-2.80)$ & 0.050 \\
\hline Post-menopausal & Yes vs. No & $1.26(0.77-2.07)$ & 0.354 & & \\
\hline Childbirth & Yes vs. No & $2.07(0.48-8.89)$ & 0.330 & & \\
\hline Tumor size $(\mathrm{cm})$ & $>3.5$ vs. $<=3.5$ & $2.05(1.25-3.34)$ & 0.004 & $1.29(0.71-2.35)$ & 0.397 \\
\hline FIGO stage & IIA2+IIA1 vs. IB1+IB2 & $2.11(1.32-3.36)$ & 0.002 & $1.76(1.02-3.02)$ & 0.041 \\
\hline $\mathrm{SCCA}(\mathrm{ng} / \mathrm{ml})$ & $>6.5$ vs. $<=6.5$ & $5.26(3.13-8.85)$ & $<0.001$ & $5.20(3.07-8.81)$ & $<0.001$ \\
\hline
\end{tabular}

Abbreviations: FIGO: International Federation of Gynecology and Obstetrics, SCCA: squamous cell carcinoma antigen

Table 5: Actual rates of para-aortic lymph node metastasis according to the number of risk factors in squamous cell carcinoma patients

\begin{tabular}{|l|c|c|}
\hline \multicolumn{1}{|c|}{ Risk factors } & Number & Positive PALN (\%) \\
\hline 0 & 153 & $8(5.2 \%)$ \\
\hline 1 & 228 & $21(9.2 \%)$ \\
\hline 2 & 213 & $47(22.1 \%)$ \\
\hline 3 & 45 & $16(35.6 \%)$ \\
\hline Total & 639 & $92(14.4 \%)$ \\
\hline
\end{tabular}

Abbreviations: PALN: para-aortic lymph node

Risk factors: 1. FIGO stage=IIA(vs. IB), 2. age $>46 \mathrm{y}, 3$. SCCA $>6.5 \mathrm{ng} / \mathrm{ml}$.

\section{Logistic analyses of PALN metastasis in SCC}

When SCC cases were analyzed, SCCA baseline level was taken into consideration (Table 4). The relative predictive factors in univariate logistic analyses were age $>46 y$, tumor size $>3.5 \mathrm{~cm}$, FIGO stage IIA, and SCCA $>6.5 \mathrm{ng} / \mathrm{ml}$. Further multivariate model indicated 3 covariates: age $>46 y$ (OR: 1.67, 95\% CI: 1.00-2.80; $p=0.050)$, FIGO stage IIA ( $v s . \mathrm{IB})(\mathrm{OR}: 1.76,95 \% \mathrm{CI}$ : $1.02-3.02 ; p=0.041$ ), and SCCA $>6.5 \mathrm{ng} / \mathrm{ml}$ (OR: 5.20 , $95 \%$ CI: $3.07-8.81 ; p<0.001)$. The positive PALN rates of patients with $0(n=153), 1(n=228), 2(n=213)$, and 3 $(n=45)$ of the above risk factors were $5.2 \%, 9.2 \%, 22.1 \%$, and $35.6 \%$, respectively (Table 5). 


\section{DISCUSSION}

In this study, we retrospectively inquired into the relationship between clinical factors and PALN metastasis in patients with stage IB1-IIA2 cervical cancer. Results showed that age, tumor size, FIGO stage, and SCCA elevation might indicate higher probability of PALN involvement. To our knowledge, this is the largest population-based analysis of para-aortic lymphadenectomy in cervical cancer.

PALN metastasis is a prognostic factor in cervical cancer [9-11]. A Gynecologic Oncology Group (GOG) study demonstrated that cervical cancer patients had worse 2-year progression-free survival dropping from 64\% down to $14 \%$ with PALN metastasis [12]. In a prospective multicenter study, 3-year overall survival (OS) of locally advanced cervical cancer patients (FIGO stage IB2-IVA) without PALN metastasis was $89 \%$, while 3-year OS of those with PALN metastasis decreased to $40 \%$ [13]. Our survival data also showed that PALN status impacted the 3-year survival rate.

Imaging is widely used in determining lymph node metastasis, but lymph node micrometastasis may not accurately be detected with CT or MRI $[14,15]$. Thus we thought it could be more appropriate and reasonable to predict PALN metastasis by combining multiple ways, but not imaging alone. A nomogram model using age, tumor size by MRI, and PET-CT result can predict LN metastasis in early stage cancer [16]. It showed a high negative predictive value of identifying low risk patients whose lymphadenectomy might be unnecessary. However, considering that the majority of their patients were stage IB1 patients $(65.9 \%)$ whose PALN rate was low, there was no specific demonstration of PALN metastasis rate and PALN prediction accuracy.

Multiple studies proved that incidence of PALN metastasis increased with advancing FIGO stage [17-
19]. In a previous study of 621 cervical cancer patients, $5 \%$ of stage IB, $16 \%$ of Stage II, and $25 \%$ of stage III were found PALN metastasis at staging laparotomy [17]. Another study of 208 cases reported the incidence of PALN metastasis in stage IB-IIB was $10-40 \%$ [20]. Result in our study showed similar ratio.

In a study by Huang et al, no PALN involvement was found in patients with tumor size $<2 \mathrm{~cm}$ or negative pelvic lymph node (PLN) metastasis [21]. Our results show $4.9 \%$ positive PALN in tumors smaller than $2 \mathrm{~cm}$, which represent $10 \%$ of our cases. With our cases with tumor size $3-5 \mathrm{~cm}$, we noticed that $3.5 \mathrm{~cm}$ was the most appropriate cutoff point statistically. However, when SCC patients were categorically analyzed, we did not find significant relevance of tumor size with PALN when tumor marker was added into the variables.

SCCA is commonly used to assist in the diagnosis and surveillance of squamous cell cancers, and serum concentration of SCCA is elevated in $28-88 \%$ of patients with SCC [22, 23]. Compared to patients without lymph node metastasis, SCCA levels before treatment were higher in patients with lymph node metastasis [24]. In a previous study by Bolger et al, when pretreatment SCCA level was $>2,>4$, and $>8.6 \mathrm{ng} / \mathrm{ml}$, the positive predictive value for lymph node metastasis was $51.4 \%, 70.0 \%$, and $100 \%$, with sensitivities of $58.1 \%, 45.2 \%$, and $22.6 \%$, respectively [25].

In the majority of cases, PALN metastasis is correlated with positive PLN, and skip metastasis is lower than $4 \%$, similar with our results $[15,20,26]$. The incidence of PALN metastasis in our total sample set was $14.0 \%$, but was $32.9 \%$ when PLN was involved, and $65.3 \%$ when common iliac LN was involved. Tsuruga et al obtained similar results, showing that positive PALN rate jumped from $9 \%$ to $60 \%$ if common iliac LN was positive [9]. One limitation of these studies, including ours, was that most pathology data was collected after the surgery.

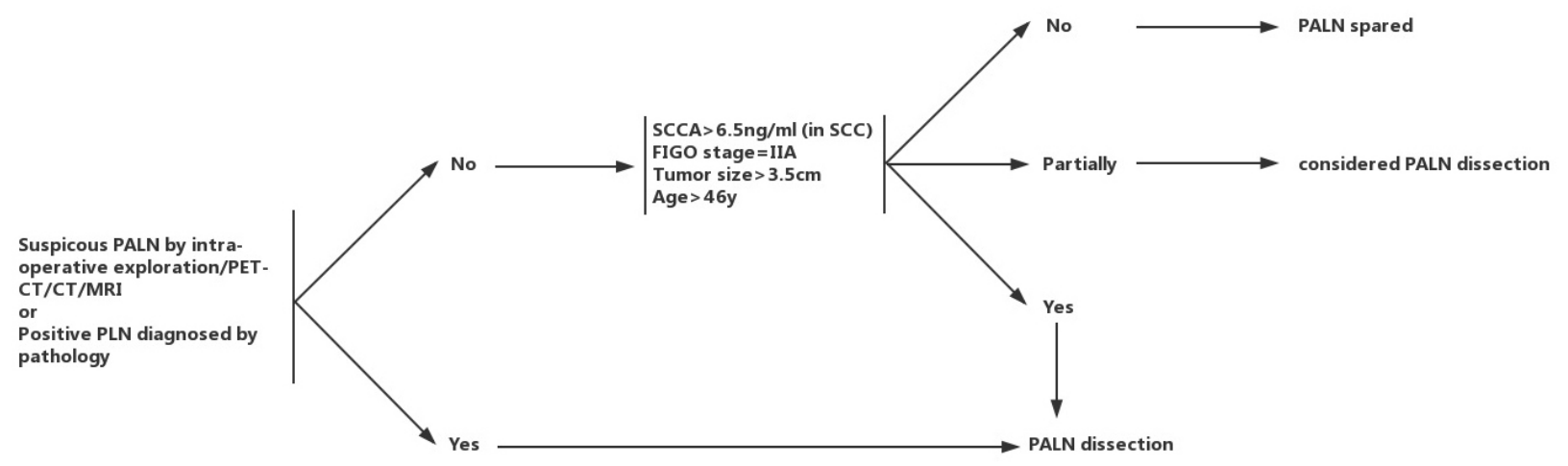

Figure 2: Flow chart of individual para-aortic lymphadenectomy in patients with stage IB1-IIA2 cervical cancer. PALN: para-aortic lymph node, PLN: pelvic lymph node (including obturator, external, internal, and common iliac lymph nodes), SCCA: squamous cell carcinoma antigen, SCC: squamous carcinoma.PALN dissection was recommended for patients under any one of the following situations: 1. suspicious PALN by intra-operative exploration/PET-CT/CT/MRI, 2. positive PLN diagnosed by pathology(e.g. frozen section, imprint smear). For the remaining patients, PALN dissection was recommended for those with FIGO stage = IIA, tumor size $>3.5 \mathrm{~cm}$, age $>46 \mathrm{y}$ and $\mathrm{SCCA}>6.5 \mathrm{ng} / \mathrm{ml}$ (in SCC). 
PLN metastasis might be hard to be accurately diagnosed during surgery, so positive pelvic LN was not designated a preoperative clinical factor in our multivariate logistic analysis.

Lv et al utilized intra-operative frozen section of common iliac LN to judge whether PALN dissection was redundant in stage IB1 and IIA1 patients, and showed higher excision accuracy of positive PALN [27]. Theoretically, frozen section of common iliac LN might be efficient to avoid unnecessary PALN dissection, but our study has explored 24 (23.8\%) positive PALN cases without common iliac LN metastasis. Other studies have observed $30 \%$ absence of common iliac LN metastasis as well $[9,21]$, suggesting it may be more appropriate to combine intra-operative frozen sections of common iliac LN with other clinical factors for predicting PALN metastasis.

According to results of this study and above analysis, we proposed a flow chart to properly identify candidates for para-aortic lymphadenectomy in patients with FIGO stage IB1-IIA2 cervical cancer (Figure 2). PALN dissection was recommended for patients under any one of the following situations: 1 . suspicious PALN by intra-operative exploration/PET-CT/CT/MRI, 2. positive PLN diagnosed by pathology(e.g. frozen section, imprint smear). For the remaining patients, PALN dissection was recommended for those with FIGO stage $=$ IIA, tumor size $>3.5 \mathrm{~cm}$, age $>46 \mathrm{y}$ and $\mathrm{SCCA}>6.5 \mathrm{ng} / \mathrm{ml}$ (in SCC). This flow chart might help physicians to distinguish highrisk patients who would benefit most from para-aortic lymphadenectomy during radical hysterectomy.

Our study had several limitations. Firstly, all our patients were recruited from a single center, which could lead to selection bias. Secondly, the present design was a retrospective study. Another possible limitation was the absence of preoperative imaging evaluation, which could also contribute to the choice of para-aortic lymphadenectomy [28]. Taken together, the efficacy of our predictors and decision strategy of PALN dissection could be further evaluated in prospective multi-center trials.

Importantly, we found that age $>46 \mathrm{y}$, tumor size $>3.5 \mathrm{~cm}$, and FIGO stage IIA ( $v s$. IB) were positively correlated with PALN metastasis in cervical cancer. In squamous cell carcinoma, age $>46 y$, FIGO stage IIA (vs. IB), and SCCA level $>6.5 \mathrm{ng} / \mathrm{ml}$ were identified as predictive factors for positive PALN. These factors can be detected easily before surgery, help to make optimum clinical decisions during radical hysterectomy.

\section{MATERIALS AND METHODS}

\section{Patients}

We conducted a retrospective study of first diagnosed cervical cancer patients who underwent PALN dissection in Fudan University Shanghai Cancer Center from January 2006 to February 2014. To eliminate the interference of confounding factors, we defined the inclusion criteria as: cervical cancer FIGO stage Ib1-IIa2, no neoadjuvant chemotherapy or radiotherapy, no second primary tumor when diagnosed, designated histological type (SCC, AC, and ACS), and complete radical surgery. After obtaining approval from our institutional review board, data was reviewed and a total of 723 patients fulfilling the above criteria were enrolled in this study. All patients signed informed written consent to therapeutic interventions (including surgery, chemotherapy, and radiotherapy if necessary).

Patients' medical records were collected, such as age, body mass index, ECOG performance status, menstrual status, baseline SCCA level, FIGO stage, tumor size, histological report, surgical procedure, ovarian metastasis, and pelvic and para-aortic lymph node status. Tumor size was measured during surgery; squamous cell carcinoma antigen (SCCA) measurement was performed using an ARCHITECT i 2000SR (Abbott, Illinois, U.S.A). The normal range is $0-1.5 \mathrm{ng} / \mathrm{ml}$.

\section{Treatment}

Gynecologic oncologists from a single center (Fudan University Shanghai Cancer Center) performed all of the radical surgeries to maintain consistency. Type III hysterectomy of Piver-Rutledge classifications was performed by laparotomy in 714 cases. The remaining 9 patients who were willing and eligible to maintain future fertility underwent abdominal radical trachelectomy as previously demonstrated [29]. All the 723 patients had systematic pelvic and para-aortic lymphadenectomy during the radical surgery procedure. Pelvic lymph nodes included the most common lymphatic pathways, such as the obturator, external, internal, and common iliac lymph nodes. PALN dissection was usually conducted along the flow of the abdominal lymphatics until reaching the inferior mesenteric artery (IMA). When infra-IMA node metastasis was suspected by preoperative evaluation or intraoperative exploration, PALNs were further resected until reaching the left renal vein.

Adjuvant treatment was used after the radical surgery when patients had high or intermediate risk prognostic factors, including positive $\mathrm{LN}$, positive margin, positive parametria, large tumor size, deep stromal invasion, and lymphovascular space invasion. Pelvic radiation at 40-50 Gy was offered with or without concurrent cisplatin-based chemotherapy individually. When PALN were involved, extended-field radiation including PLN and PALN was recommended. Platinumbased chemotherapy alone was occasionally used in some specific patients who rejected radiotherapy. 


\section{Statistical methods}

Survival curves were done by the Kaplan-Meier method and analyzed by the log-rank test. The $\chi^{2}$ test was used to assess differences in the distributions of demographic characteristics between positive PALN cases and negative PALN cases. The association between relevant factors and PALN metastasis was assessed by odds ratio (OR) and 95\% confidence interval (CI). OR values were calculated by both univariate and multivariate logistic regression models, which was applied using stepwise backward elimination until only significant $(p<$ 0.05 ) variables remained in multivariate survival analysis. All statistical analyses were performed using SPSS version 13.0 statistical software (SPSS Inc., Chicago, IL). A $p$ value of $<0.05$ was considered statistically significant.

\section{Authors' contributions}

Xiaotian Han and Hao Wen performed the majority of the project, analyzed the data, and drafted the manuscript. Xiaohua Wu conceived the project and supervised the research. All authors participated in the coordination of the study and approved the final manuscript.

\section{ACKNOWLEDGMENTS}

We are grateful to the staff of the Department of Gynecologic Oncology of Fudan University Shanghai Cancer Center for performing the surgeries, and the staff of the Department of Pathology for specimen identification.

\section{CONFLICTS OF INTEREST} interests.

The authors declare that they have no competing

\section{FUNDING}

The authors declare that they have no funding.

\section{REFERENCES}

1. Torre LA, Bray F, Siegel RL, Ferlay J, Lortet-Tieulent J, Jemal A. Global cancer statistics, 2012. CA Cancer J Clin. 2015; 65:87-108.

2. Chen W, Zheng R, Baade PD, Zhang S, Zeng H, Bray F, Jemal A, Yu XQ, He J. Cancer statistics in China, 2015. CA Cancer J Clin. 2016; 66:115-132.

3. Ditto A, Martinelli F, Lo Vullo S, Reato C, Solima E, Carcangiu M, Haeusler E, Mariani L, Lorusso D, Raspagliesi F. The role of lymphadenectomy in cervical cancer patients: the significance of the number and the status of lymph nodes removed in 526 cases treated in a single institution. Ann Surg Oncol. 2013; 20:3948-3954.

4. Gien LT, Covens A. Lymph node assessment in cervical cancer: prognostic and therapeutic implications. Journal of surgical oncology. 2009; 99:242-247.

5. Wui-Jin Koh, Greer BE, Abu-Rustum NR, Apte SM. (2016). NCCN Clinical PracticeGuidelines in Oncology: Cervical cancer, V.1.2016. USA: National Comprehensive Cancer Network: http://www.nccn.org/professionals/ physician_gls/f_guidelines.asp\#cervical).

6. Hwang L, Bailey A, Lea J, Albuquerque K. Para-aortic nodal metastases in cervical cancer: a blind spot in the International Federation of Gynecology and Obstetrics staging system: current diagnosis and management. Future Oncol. 2015; 11:309-322.

7. Choi HJ, Ju W, Myung SK, Kim Y. Diagnostic performance of computer tomography, magnetic resonance imaging, and positron emission tomography or positron emission tomography/computer tomography for detection of metastatic lymph nodes in patients with cervical cancer: meta-analysis. Cancer science. 2010; 101:1471-1479.

8. Leblanc E, Katdare N, Narducci F, Bresson L, Gouy S, Morice P, Ferron G, Querleu D, Martinez A. Should Systematic Infrarenal Para-aortic Dissection Be the Rule in the Pretherapeutic Staging of Primary or Recurrent Locally Advanced Cervix Cancer Patients With a Negative Preoperative Para-aortic PET Imaging? Int J Gynecol Cancer. 2016; 26:169-175.

9. Tsuruga T, Fujimoto A, Kawana K, Mori M, Hasumi Y, Kino N, Tomio K, Miura S, Tanikawa M, Sone K, Miyamoto Y, Ikeda Y, Kojima S, et al. Radical hysterectomy with or without para-aortic lymphadenectomy for patients with stage IB2, IIA2, and IIB cervical cancer: outcomes for a series of 308 patients. Int J Clin Oncol. 2016; 21:359-66.

10. Chereau E, Feron JG, Ballester M, Coutant C, Bezu C, Rouzier R, Touboul E, Darai E. Contribution of pelvic and para-aortic lymphadenectomy with sentinel node biopsy in patients with IB2-IIB cervical cancer. Br J Cancer. 2012; 106:39-44.

11. Stryker JA, Mortel R. Survival following extended field irradiation in carcinoma of cervix metastatic to para-aortic lymph nodes. Gynecol Oncol. 2000; 79:399-405.

12. Grigsby PW, Heydon K, Mutch DG, Kim RY, Eifel P. Long-term follow-up of RTOG 92-10: cervical cancer with positive para-aortic lymph nodes. Int J Radiat Oncol Biol Phys. 2001; 51:982-987.

13. Gouy S, Morice P, Narducci F, Uzan C, Martinez A, Rey A, Bentivegna E, Pautier P, Deandreis D, Querleu D, Haie-Meder C, Leblanc E. Prospective multicenter study evaluating the survival of patients with locally advanced cervical cancer undergoing laparoscopic para-aortic lymphadenectomy before chemoradiotherapy in the era of positron emission tomography imaging. J Clin Oncol. 2013; 31:3026-3033 
14. Zand B, Euscher ED, Soliman PT, Schmeler KM, Coleman RL, Frumovitz M, Jhingran A, Ramondetta LM, Ramirez PT. Rate of para-aortic lymph node micrometastasis in patients with locally advanced cervical cancer. Gynecol Oncol. 2010; 119:422-425.

15. Bats AS, Mathevet P, Buenerd A, Orliaguet I, Mery E, Zerdoud S, Le Frere-Belda MA, Froissart M, Querleu D, Martinez A, Leblanc E, Morice P, Darai E, et al. The sentinel node technique detects unexpected drainage pathways and allows nodal ultrastaging in early cervical cancer: insights from the multicenter prospective SENTICOL study. Annals of surgical oncology. 2013; 20:413-422.

16. Kim DY, Shim SH, Kim SO, Lee SW, Park JY, Suh DS, Kim JH, Kim YM, Kim YT, Nam JH. Preoperative nomogram for the identification of lymph node metastasis in early cervical cancer. Br J Cancer. 2014; 110:34-41.

17. Berman ML, Keys H, Creasman W, DiSaia P, Bundy B, Blessing J. Survival and patterns of recurrence in cervical cancer metastatic to periaortic lymph nodes (a Gynecologic Oncology Group study). Gynecologic oncology. 1984; 19:8-16.

18. Michel G, Morice P, Castaigne D, Leblanc M, Rey A, Duvillard P. Lymphatic spread in stage Ib and II cervical carcinoma: anatomy and surgical implications. Obstet Gynecol. 1998; 91:360-363.

19. Grigsby PW, Siegel BA, Dehdashti F. Lymph node staging by positron emission tomography in patients with carcinoma of the cervix. J Clin Oncol. 2001; 19:3745-3749.

20. Sakuragi N, Satoh C, Takeda N, Hareyama H, Takeda M, Yamamoto R, Fujimoto T, Oikawa M, Fujino T, Fujimoto $\mathrm{S}$. Incidence and distribution pattern of pelvic and paraaortic lymph node metastasis in patients with Stages IB, IIA, and IIB cervical carcinoma treated with radical hysterectomy. Cancer. 1999; 85:1547-1554.

21. Huang H, Liu J, Li Y, Wan T, Feng Y, Li Z, Huang Q. Metastasis to deep obturator and para-aortic lymph nodes in 649 patients with cervical carcinoma. Eur J Surg Oncol. 2011; 37:978-983.
22. Gadducci A, Tana R, Cosio S, Genazzani AR. The serum assay of tumour markers in the prognostic evaluation, treatment monitoring and follow-up of patients with cervical cancer: a review of the literature. Critical reviews in oncology/hematology. 2008; 66:10-20.

23. Zhi W, Ferris D, Sharma A, Purohit S, Santos C, He M, Ghamande S, She JX. Twelve serum proteins progressively increase with disease stage in squamous cell cervical cancer patients. International journal of gynecological cancer. 2014; 24:1085-1092.

24. Kim YT, Yoon BS, Kim JW, Kim SH, Kwon JY, Kim JH. Pretreatment levels of serum squamous cell carcinoma antigen and urine polyamines in women with squamous cell carcinoma of the cervix. International journal of gynaecology and obstetrics. 2005; 91:47-52.

25. Bolger BS, Dabbas M, Lopes A, Monaghan JM. Prognostic value of preoperative squamous cell carcinoma antigen level in patients surgically treated for cervical carcinoma. Gynecologic oncology. 1997; 65:309-313.

26. Hackett TE, Olt G, Sorosky JI, Podczaski E, Harrison TA, Mortel R. Surgical predictors of para-aortic metastases in early-stage cervical carcinoma. Gynecologic oncology. 1995; 59:15-19.

27. Lv X, Chen L, Yu H, Zhang X, Yan D. Intra-operative frozen section analysis of common iliac lymph nodes in patients with stage IB1 and IIA1 cervical cancer. Arch Gynecol Obstet. 2012; 285:811-816.

28. Boughanim M, Leboulleux S, Rey A, Pham CT, Zafrani Y, Duvillard P, Lumbroso J, Haie-Meder C, Schlumberger M, Morice P. Histologic results of paraaortic lymphadenectomy in patients treated for stage IB2/ II cervical cancer with negative $[18 \mathrm{~F}]$ fluorodeoxyglucose positron emission tomography scans in the para-aortic area. J Clin Oncol. 2008; 26:2558-2561.

29. Li J, Li Z, Wang H, Zang R, Zhou Y, Ju X, Ke G, Wu X. Radical abdominal trachelectomy for cervical malignancies: surgical, oncological and fertility outcomes in 62 patients. Gynecologic Oncology. 2011; 121:565-570. 